\title{
Tensor Product and Semi-stability: Four Variations on a Theme
}

Marco Maculan (Université Pierre et Marie Curie, Paris, France)

Chevalley proved that in characteristic 0 the tensor product of semi-simple representations is semi-simple. This result has analogues in rather diverse contexts: three of them are presented here in independent sections, focusing on the differences of the frameworks and the similarities of the proofs. Algebraic groups will play a crucial role, sometimes in unexpected ways.

\section{Representations}

Let $G$ be a group and $k$ a field. In this note, a representation of $G$ is a finite-dimensional $k$-vector space $V$ together with a group homomorphism $\rho: G \rightarrow \operatorname{GL}(V)$.

\section{Semi-simple representations}

A representation is said to be:

- irreducible if there are exactly two sub-vector spaces of $V$ stable under the action of $G$ : the zero subspace 0 and the whole vector space $V$. In particular the zero representation is not considered to be irreducible.

- semi-simple if it can be decomposed into irreducible ones: there are irreducible sub-representations $V_{1}, \ldots, V_{n}$ of $V$ such that $V=V_{1} \oplus \cdots \oplus V_{n}$. This is equivalent to saying that for every $G$-stable subspace $W$ of $V$ there is a $G$-stable supplement $W^{\prime}$.

Theorem 1 (Chevalley [8], p. 88). Suppose char $(k)=0$. The tensor product of semi-simple representations $V_{1}, V_{2}$ of $G$ is semi-simple.

The proof of Chevalley's theorem is a beautiful application of the theory of linear algebraic groups (that is, groups of matrices defined by polynomial equations), even though the group $G$ may not at all be of this form.

Indeed, in order to prove theorem 1, one may suppose that the field $k$ is algebraically closed and look at $\operatorname{GL}\left(V_{1}\right), \operatorname{GL}\left(V_{2}\right)$ as algebraic groups.

Then one can suppose $G$ to be itself a linear algebraic group. For, it suffices to take the Zariski-closure $\bar{G}$ of the image of $G$ in $\operatorname{GL}\left(V_{1}\right) \times \operatorname{GL}\left(V_{2}\right)$, namely the set of points

$$
x \in \mathrm{GL}\left(V_{1}\right) \times \mathrm{GL}\left(V_{2}\right)
$$

such that $f(x)=0$ for all polynomial functions $f$ vanishing identically on the image of $G$. The semi-simplicity of the representation $V_{1} \otimes_{k} V_{2}$ is equivalent for $G$ and $\bar{G}$, because it is a condition that can be expressed as the vanishing of some polynomials.

Now the theory of linear algebraic groups applies: there is an algebraic subgroup $\operatorname{rad}^{u}(G)$ of $G$ called the unipotent radical which is connected, unipotent (meaning that all the eigenvalues of its elements are 1), normal and contains any other subgroup of $G$ with these three properties. The unipotent radical controls the semi-simplicity of the representations of $G$ :

Theorem 2 (Weyl [33]). Suppose char $(k)=0$. An algebraic ${ }^{1}$ representation of a linear algebraic group $G$ is semi-simple if and only if every element of the unipotent radical acts as the identity on $V$.

Applying the preceding fact, the proof of Chevalley's theorem is easily achieved: since the representations $V_{1}$ and $V_{2}$ are supposed to be semi-simple, an element $g$ of the unipotent radical of $G$ acts as the identity on $V_{1}$ and $V_{2}$. Therefore $g$ operates trivially on $V_{1} \otimes_{k} V_{2}$ too.

\section{Reductive groups}

Weyl's theorem is usually formulated as follows: an algebraic representation of a reductive group, i.e. a linear algebraic group whose unipotent radical is trivial, is semi-simple.

Examples of reductive groups are $\mathrm{GL}_{n}, \mathrm{SL}_{n}, \mathrm{SO}_{n}, \mathrm{Sp}_{2 n}$. In general, a reductive group is the extension of a semi-simple group by a product of copies of the multiplicative group $k^{*}$, the algebraic equivalent of $\mathbb{C}^{*}$. An example of a non-reductive group is the additive group $k^{2}$, which corresponds to the complex Lie group $\mathbb{C}$.

Over the complex numbers, a reductive group $G$ can be seen also as a complex Lie group. Therefore two topologies cohabit in it: the one coming from the topology of $\mathbb{C}$ (the "usual topology"), and the Zariski one (which is coarser). This is true for every algebraic group, but there is a topological property which is specific to reductive groups: they contain subgroups which are dense for the Zariski topology and compact for the usual one.

For instance, a Zariski-dense compact subgroup of $\mathrm{GL}_{n}(\mathbb{C})$ is the group $\mathbb{U}(n)$ of unitary matrices; on the other hand, the unique compact subgroup of $\mathbb{C}$ is the trivial one (which is not Zariski-dense).

For a semi-simple group $G$ one uses É. Cartan's classification of semi-simple Lie algebras to construct a Zariskidense compact subgroup $K$ : indeed the Killing form on Lie $G$ is non-degenerate, and $K$ corresponds to a maximal real Lie sub-algebra on which the form is negative definite.

\footnotetext{
${ }^{1}$ This means that the map $G \rightarrow \operatorname{GL}(V)$ is polynomial in the coefficients of the matrices of $G$.

${ }^{2}$ The additive group $k$ can be seen as a subgroup of $\mathrm{GL}_{2}(k)$ through the embedding $a \longmapsto\left(\begin{array}{ll}1 & a \\ 0 & 1\end{array}\right)$
} 


\section{Weyl's Unitarian Trick}

Let us go back to the proof of theorem 2, in the second reformulation I gave above. Quite often in algebraic geometry the characteristic 0 hypothesis is the shade of transcendental techniques, that is, analysis on real or complex numbers. Theorem 2 is an example of this phenomenon.

Indeed, in order to prove it one reduces to the case where $k$ is $\mathbb{C}$ : this is possible because the polynomial equations defining $G$ and its representation $G \rightarrow \operatorname{GL}(V)$ involve only finitely many terms that are transcendental over $\mathbb{Q}$.

Given a $G$-stable subspace $W$ of $V$, finding a $G$-stable supplement amounts to construct a $G$-equivariant linear projection $V \rightarrow W$.

Here comes a remarkable argument, refined gradually by Hilbert, Hurwitz and Weyl, who at last named it Unitarian Trick. Pick one linear projection $\pi: V \rightarrow W$ and average it on a Zariski-dense compact subgroup $K$ of $G$ :

$$
\tilde{\pi}(v):=\int_{K} g \pi\left(g^{-1} v\right) d \mu(g), \quad(v \in V),
$$

where $\mu$ is the Haar measure of total mass 1 on $K$. The function $\tilde{\pi}$ is equivariant for the action of $K$, but by Zariski-density of $K$, the projection $\tilde{\pi}$ is also $G$-equivariant.

A motto summarizing the previous proof might be: the subgroup $K$ is small for the usual topology (which permits to integrate), but big enough to control the representations of $G$ (which depends only on the Zariski topology).

\section{Characteristic $p>0$}

Over a field $k$ of characteristic $p>0$ (assumed algebraically closed, for simplicity), both theorems 1 and 2 are false.

On the positive side, every representation $V$ of an algebraic group $G$ is semi-simple in the following cases:

- $G$ is a finite group whose order is prime to $p$ : given a $G$ stable subspace $W \subset V$ and a projection $\pi: V \rightarrow W$, the function

$$
\tilde{\pi}(v):=\frac{1}{\# G} \sum_{g \in G} g \varphi\left(g^{-1} v\right),
$$

is $G$-equivariant;

- $G$ is the multiplicative group $k^{*}$ : in this case $V$ decomposes as

$$
V=\bigoplus_{a \in \mathbb{Z}} V_{a}
$$

where $V_{a}$ is the subspace where $t \in k^{*}$ acts by $t^{a}$.

Nagata [11, Théorème IV.3.3.6] shows that there are no other possibilities: given $G$ an algebraic group such that every representation is semi-simple, the identity component $G^{0}$ is isomorphic to $\mathbb{G}_{m}^{r}$ and $G / G^{0}$ has order prime to $p$.

Concerning theorem 1 one has the following:

Example 3. Let $k$ be a field characteristic $p>0$ and $V=k^{2}$. Consider the $k$-vector space $V(d)$ of homogeneous polynomials of degree $d$ on $V$ : it is of dimension $d+1$, a basis being given by the monomials

$$
x^{d}, x^{d-1} y, \ldots, x y^{d-1}, y^{d} .
$$

The group $G=\mathrm{SL}_{2}(k)$ acts on $V(d)$ through the contragradient representation: explicitly, for a polynomial $f(x, y)$ and an invertible matrix

$$
g=\left(\begin{array}{ll}
\alpha & \beta \\
\gamma & \delta
\end{array}\right)
$$

with $\alpha, \beta, \gamma, \delta \in k$ such that $\alpha \delta-\beta \gamma=1$,

$$
(g f)(x, y)=f\left(g^{-1}(x, y)\right)=f(\delta x-\beta y,-\gamma x+\alpha y) .
$$

The representation $V(d)$ is irreducible for $d=1, \ldots, p-1$. For $d=p$ the map $\varphi: V(1) \rightarrow V(p)$, associating to a linear form $f$ its $p$-th power $f^{p}$, is linear, injective and $G$-equivariant. The image of $\varphi$ is the kernel of the map $\psi: V(p) \rightarrow V(p-2)$ given by

$$
f \longmapsto \frac{1}{y} \frac{\partial f}{\partial x}
$$

The exact sequence of representations

$$
0 \longrightarrow V(1) \stackrel{\varphi}{\longrightarrow} V(p) \stackrel{\psi}{\longrightarrow} V(p-2) \longrightarrow 0,
$$

obtained in this way does not split if the field $k$ has at least 3 elements. In particular $V(p)$ is not semi-simple. If $d_{1}, \ldots, d_{n}$ are integers ranging between 1 and $p-1$ such that their sum is $p$, the multiplication map $f_{1} \otimes \cdots \otimes f_{n} \mapsto f_{1} \cdots f_{n}$ induces a surjection $W:=V\left(d_{1}\right) \otimes \cdots \otimes V\left(d_{n}\right) \rightarrow V(p)$. It follows that $W$ is not semi-simple.

However Serre shows that these problems do not occur as soon as the dimension is small enough:

Theorem 4 (Serre [29]). Let $W_{1}, \ldots, W_{m}$ be semi-simple representations of a group $G$ on a field of characteristic $p>0$. If

$$
\sum_{i=1}^{m} \operatorname{dim} W_{i}<p+m,
$$

then $W_{1} \otimes \cdots \otimes W_{m}$ is semi-simple.

According to Example 3 the condition in the theorem above is sharp. Various generalizations of the result of Serre can be found in $[30,10,2]$.

\section{Vector bundles on Riemann surfaces}

\section{Representations of the fundamental group}

Let $X$ be a compact Riemann surface of genus $g$ and $\tilde{X}$ its universal covering. The fundamental group $\pi_{1}(X, x)$ (with respect to a base point $x$ ) is generated by $2 g$ loops $a_{1}, \ldots, a_{g}, b_{1}, \ldots, b_{g}$ satisfying the relation

$$
a_{1} b_{1} a_{1}^{-1} b_{1}^{-1} \cdots a_{g} b_{g} a_{g}^{-1} b_{g}^{-1}=1 .
$$

To a representation $\rho: \pi_{1}(X, x) \rightarrow \mathrm{GL}_{r}(\mathbb{C})$ of the fundamental group, one associates the vector bundle $E(\rho)$ of rank $r$ on $X$ obtained as the quotient of $\tilde{X} \times \mathbb{C}^{r}$ through the action

$$
g(\tilde{x}, v)=(g \tilde{x}, \rho(g) v) .
$$

Theorem 5 (Narasimhan-Seshadri [25]). Suppose $\rho$ preserves a hermitian norm on $\mathbb{C}^{r}$. Then:

1. $E(\rho)$ is a direct sum of simple bundles, and $E(\rho)$ is simple if and only $\rho$ is irreducible;

2. if $E(\rho)$ is simple, then it does not admit non-zero global holomorphic sections;

3. $\operatorname{deg} E(\rho)=0$ and $E(\rho)$ is semi-stable: $\operatorname{deg} F \leq 0$ for every sub-vector bundle $F$ of $E(\rho)$.

Moreover, every stable vector bundle of degree 0 arises from a unitary representation of the fundamental group of $X$.

In the previous statement, a vector bundle $E$ is said to be: - simple if the only endomorphisms of $E$ are homotheties; 
- semi-stable (resp. stable) if for every sub-vector bundle $F$ of $E$ different from 0 and $E$,

$$
\frac{\operatorname{deg} F}{\operatorname{rk} F} \leq \frac{\operatorname{deg} E}{\operatorname{rk} E}, \quad(\text { resp. }<) .
$$

The number $\mu(E):=\frac{\operatorname{deg} E}{\operatorname{rk} E}$ is called the slope of $E$.

Remark that a simple bundle $E$ cannot be written as the direct sum of two proper sub-bundles.

Statements (1) and (2) are consequences of the following isomorphism, for unitary representations $\rho_{i}: \pi_{1}(X, x) \rightarrow$ $\mathrm{GL}\left(V_{i}\right)$ and $i=1,2$,

$$
\operatorname{Hom}_{\pi_{1}(X, x)}\left(V_{1}, V_{2}\right) \stackrel{\sim}{\longrightarrow} \operatorname{Hom}\left(E\left(\rho_{1}\right), E\left(\rho_{2}\right)\right) .
$$

Statement (1) is obtained thanks to Weyl's Unitarian Trick, by decomposing the representation $\rho$ into its irreducible components. In order to get a taste of how properties of the representation $\rho$ transfer to those of the vector bundle $E(\rho)$, let me sketch the proof of (3).

The line bundle $L:=\bigwedge^{r} E(\rho)$ is associated to the determinant representation $\operatorname{det} \rho: \pi_{1}(X, x) \rightarrow \mathbb{C}^{\times}$of $\rho$, which is unitary. If the degree of $L$ were positive, by the Riemann-Roch theorem, some positive enough multiple $L^{\otimes d}$ of $L$ would admit non-zero global sections: this would contradict (2), as $L^{\otimes d}$ is indecomposable. One concludes by applying the same reasoning to the determinant of the representation $\rho^{*}$ contragradient to $\rho$.

Take $F$ to be a sub-vector bundle of $E(\rho)$ and, arguing by contradiction, assume that the degree of $F$ is $>0$. One can further suppose that $F$ is a line bundle: if $s$ denotes the rank of $F$, the $s$-th exterior power $\bigwedge^{s} F$ is a sub-line bundle of the vector bundle $\bigwedge^{s} E(\rho)$, which is associated to the representation $\bigwedge^{s} \rho$.

As before, by the Riemann-Roch theorem, there is a positive integer $d \geq 1$ such that $L^{\otimes d}$ has non-zero sections. Decompose the unitary representation $\rho^{\otimes d}$ into irreducible ones $\rho_{1}, \ldots, \rho_{n}$. This corresponds to writing $E(\rho)^{\otimes d}$ as the sum of the simple vector bundles $E\left(\rho_{i}\right)$. The projection of $L$ onto $E\left(\rho_{i}\right)$ has to be zero for all $i=1, \ldots, n$ : otherwise $E\left(\rho_{i}\right)$ would have non-zero sections, which is impossible according to (2). This implies that $F$ is the trivial bundle, contradicting the hypothesis of having positive degree.

The results of Narasimhan-Seshadri have been for quite a long time the only tool to prove the following result:

Theorem 6. Let E, F be semi-stable vector bundles on $X$. Then $E \otimes F$ is semi-stable.

The key situation is when $E$ and $F$ are stable of degree 0 : if this is the case, thanks to the theorem of NarasimhanSeshadri, $E$ and $F$ are associated to unitary representations $\rho_{E}, \rho_{F}$ of the fundamental group. The tensor product representation $\rho_{E} \otimes \rho_{F}$ is unitary, thus $E \otimes F$ is semi-stable.

\section{Characteristic $p>0$}

On a field of positive characteristic, compact Riemann surfaces are replaced by smooth projective curves.

To fix ideas, let $f \in \mathbb{F}_{p}\left[x_{0}, x_{1}, x_{2}\right]$ be an irreducible homogeneous polynomial and consider the locus in $\mathbb{P}^{2}\left(\overline{\mathbb{F}}_{p}\right)$ where it vanishes:

$$
X=\left\{\left[x_{0}: x_{1}: x_{2}\right] \in \mathbb{P}^{2}\left(\overline{\mathbb{F}}_{p}\right): f\left(x_{0}, x_{1}, x_{2}\right)=0\right\} .
$$

Suppose that $X$ does not have singular points: this means that for every point $x \in X$, some partial derivative $\frac{\partial f}{\partial x_{i}}$ does not vanish at $x$.

The genus $g$ of $X$ can be computed as for Riemann surfaces: if $f$ is of degree $d$, then

$$
g=\frac{(d-1)(d-2)}{2} .
$$

However $X$ carries something that a Riemann surface does not, the Frobenius endomorphism. It is the map sending a point $\left[x_{0}: x_{1}: x_{2}\right] \in X$ to $\left[x_{0}^{p}: x_{1}^{p}: x_{2}^{p}\right]$. Note that the latter point still belongs to $X$ because

$$
f\left(x_{0}^{p}, x_{1}^{p}, x_{2}^{p}\right)=f\left(x_{0}, x_{1}, x_{2}\right)^{p}=0,
$$

(one uses here that $f$ has coefficients in $\mathbb{F}_{p}$ ).

There is no obvious way to port unitary representations of the fundamental group into this context. Rather, one takes the point of view of semi-stable vector bundles, whose definition can be translated word by word. Yet the statement analogous to theorem 6 is false and, as one can guess, examples come from symmetric powers:

Example 7. Let $E$ be a vector bundle on $X$ and consider its $i$-th symmetric power $\operatorname{Sym}^{i} E$.

The naive idea of embedding $E$ into $\operatorname{Sym}^{p} E$ by raising sections to the $p$-th power does not work this time: the map $E \rightarrow \operatorname{Sym}^{p} E,(x, s) \mapsto\left(x, s^{p}\right)$, where $x$ is a point of $X$ and $s$ is a section of $E$ over $x$, is not a bundle map. Nonetheless it induces an inclusion of the pull-back $\operatorname{Fr}_{X}^{*} E$ of $E$ (the so-called Frobenius twist) as a sub-vector bundle of $\operatorname{Sym}^{p} E$.

If the genus of $X$ is at least 2, there are plenty of semistable vector bundles on $X$ such that their Frobenius twist is not semi-stable ${ }^{3}$ : for such a vector bundle $E$, the above discussion shows that $\operatorname{Sym}^{p} E$ is not semi-stable.

There is also an analogue of Serre's theorem for semistable vector bundles (Balaji-Parameswaran [3], IlangovanMehta-Parameswaran [18]):

Theorem 8. Let $E_{1}, \ldots, E_{n}$ be semi-stable vector bundles on X. If

$$
\sum_{i=1}^{n} \mathrm{rk} E_{i}<p+n,
$$

then $E_{1} \otimes \cdots \otimes E_{n}$ is semi-stable.

\section{Back to characteristic 0}

In view of the preceding example, an algebraic proof of theorem 6 has to break down in positive characteristic. Let me detail the one discovered by Ramanan-Ramanathan [26]: it is particularly interesting from our point of view because it makes intervene algebraic groups (where a priori there are none).

Let $V_{1}$ and $V_{2}$ be semi-stable vector bundles on a compact Riemann surface $X$, and let $W$ be a sub-vector bundle of the

\footnotetext{
${ }^{3}$ For instance, consider the push-forward $V:=\left(\operatorname{Fr}_{X}\right)_{*} O_{X}$ of the trivial bundle $O_{X}$. A simple computation shows

$$
\operatorname{deg} V=(p-1)(g-1)>0 .
$$

By the Harder-Narasimhan filtration, $V$ contains a semi-stable vector bundle $E$ of positive degree. The kernel $K$ of the induced map $\operatorname{Fr}_{X}^{*} E \rightarrow O_{X}$ has degree $\geq \operatorname{deg} \operatorname{Fr}_{X}^{*} E$. Having smaller rank, the slope of $K$ is bigger than the slope of $\operatorname{Fr}_{X}^{*} E$.
} 
is easy to obtain when $W$ is "special" (for instance if $W$ is of the form $W_{1} \otimes W_{2}$ for sub-vector bundles $W_{i} \subset V_{i}$ ) or if it is "generic" (meaning that its intersection with some filtration has small dimension).

This heuristics is made precise by Ramanan-Ramanathan: replacing the locutions "special" and "generic" respectively by unstable and semi-stable in the sense of Geometric Invariant Theory (GIT) permits to derive (1) in both cases. The algebraic group that enters into play is $\mathrm{GL}_{r_{1}, K} \times \mathrm{GL}_{r_{2}, K}$ where $K$ is the field of meromorphic functions on $X$ and $r_{i}$ the rank of $V_{i}$.

What goes wrong in characteristic $p$ is the characterization of semi-stability they use (the Hilbert-Mumford criterion [17, 24], in the version of Kempf [20] and Rousseau [27]) that needs the field $K$ to be perfect.

If $X$ is a compact Riemann surface, then $K$ is perfect because of characteristic 0 . If $X$ is a smooth projective curve over a field $k$ of characteristic $p, K$ is a finite extension of the field $k(t)$ of rational functions in one variable, thus imperfect.

\section{Weakly admissible isocrystals}

\section{Counting points with eigenvalues}

Let $f\left(x_{1}, \ldots, x_{n}\right)$ be a polynomial with integral coefficients. The set of its solutions modulo $p$,

$$
V\left(\mathbb{F}_{p}\right):=\left\{x=\left(x_{1}, \ldots, x_{n}\right) \in \mathbb{F}_{p}^{n}: f(x)=0\right\},
$$

is finite. What can we say about its cardinality?

There is a fruitful way to look at solutions modulo $p$. Consider the affine variety defined by $f$,

$$
V:=\left\{x \in \overline{\mathbb{F}}_{p}^{n}: f(x)=0\right\} .
$$

As an element $a \in \overline{\mathbb{F}}_{p}$ belongs to $\mathbb{F}_{p}$ if and only if $a^{p}=a$, a point $\left(x_{1}, \ldots, x_{n}\right) \in V$ lies in $V\left(\mathbb{F}_{p}\right)$ if and only if $x_{i}^{p}=x_{i}$ for all $i$. In other words, $V\left(\mathbb{F}_{p}\right)$ is the set of fixed points of the Frobenius endomorphism of $V$ (i.e. the map raising to the $p$-th power the coordinates of the points of $X$ ).

A brilliant idea of Weil was to relate this point of view with the following form of the "Lefschetz fixed point theorem", known as the Lefschetz-Hopf theorem:

Theorem 9. Let $X$ be a compact manifold and $F: X \rightarrow X$ a continuous map. If the set $\operatorname{Fix}(F)$ of fixed points of $F$ is finite, then

$$
\sum_{x \in \operatorname{Fix}(F)} i(x, F)=\sum_{i \geq 0}(-1)^{i} \operatorname{Tr}\left(F_{*} \mid \mathrm{H}_{i}(X, \mathbb{Q})\right),
$$

where $i(x, F)$ is the index of $F$ at a fixed point $x$ and $\mathrm{H}_{i}(X, \mathbb{Q})$ the $i$-th rational homology group.

Weil conjectured that if there were a cohomology theory that behaved well enough (called in this day and age "Weil cohomology theory") one could compute the number of rational points by means of an analogue of the Lefschetz fixed point theorem.

Constructing such a cohomology theory has been one of the driving forces behind the work of Grothendieck and his school. The results in SGA 4 and 5 say that there is actually one for each prime $\ell \neq p$ : the étale $\ell$-adic cohomology, with coefficients in the field $\mathbb{Q}_{\ell}$ of $\ell$-adic numbers. Grothendieck in particular was able to prove an analogue of Lefschetz's fixed point theorem:
Theorem 10 (Grothendieck's trace formula [16]). Let $X$ be a

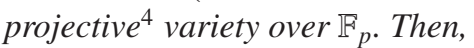

$$
\# X\left(\mathbb{F}_{p}\right)=\sum_{i \geq 0}(-1)^{i} \operatorname{Tr}\left(\operatorname{Fr}_{X} \mid \mathrm{H}_{e t}^{i}\left(X, \mathbb{Q}_{\ell}\right)\right),
$$

where $X\left(\mathbb{F}_{p}\right)$ is the set of points of $X$ having coordinates in $\mathbb{F}_{p}$.

Let us go back to counting the points of $V\left(\mathbb{F}_{p}\right)$. The preceding theorem suggests to pass to the projective closure of $V$ : consider the homogeneous polynomial associated to $f$,

$$
\tilde{f}\left(x_{0}, \ldots, x_{n}\right):=x_{0}^{d} f\left(x_{1} / x_{0}, \ldots, x_{n} / x_{0}\right),
$$

where $d$ is the degree of $f$, and

$$
X:=\left\{x=\left[x_{0}: \cdots: x_{n}\right] \in \mathbb{P}^{n}\left(\overline{\mathbb{F}}_{p}\right): \tilde{f}(x)=0\right\} .
$$

With this at hand, the philosophy can be restated as follows: in order to compute the number of rational points of $X$ (i.e. those with coordinates in $\mathbb{F}_{p}$ ), one has to estimate the size of the eigenvalues of the Frobenius acting on the $\ell$-adic étale cohomology.

If $X$ is non-singular, the following facts are consequences of Deligne's proof of the last of Weil's conjectures (called Riemann hypothesis):

1. the set of eigenvalues of $\operatorname{Fr}_{X}$ on $\mathrm{H}_{e t}^{i}\left(X, \mathbb{Q}_{\ell}\right)$ does not depend on $\ell$;

2. an eigenvalue of $\operatorname{Fr}_{X}$ on $\mathrm{H}_{e t}^{i}\left(X, \mathbb{Q}_{\ell}\right)$ is an algebraic integer, which is not divisible by $\ell$ and whose complex absolute value is $\sqrt{p^{i}}$ (with respect to any complex embedding).

\section{Divisibility properties}

A question left aside is the order of divisibility by $p$ of the eigenvalues of the Frobenius, or in other words, their $p$-adic valuation. A first result of this kind is the following:

Theorem 11 (Chevalley-Warning [28]). Suppose $\operatorname{deg} f<n$. Then $\# V\left(\mathbb{F}_{p}\right)$ is divisible by $p$.

In order to study such a question it seems appropriate to ask the cohomology theory to produce $\mathbb{Q}_{p}$-vector spaces. Unfortunately $p$-adic étale cohomology does not work as one would like and one has to consider crystalline cohomology.

From now on suppose $X$ non-singular and that $f$ is not divisible by $p$. The polynomial $f$ we started with, as well as its homogeneization $\tilde{f}$, have integral coefficients. Consider the projective variety, defined over $\mathbb{Q}$,

$$
Y:=\left\{x=\left[x_{0}: \cdots: x_{n}\right] \in \mathbb{P}^{n}(\overline{\mathbb{Q}}): \tilde{f}(x)=0\right\} .
$$

As a consequence of Berthelot's results on crystalline cohomology, the algebraic de Rham cohomology groups of $Y$, or better said their extension to $\mathbb{Q}_{p}$,

$$
\mathrm{H}_{\mathrm{dR}}^{q}\left(X / \mathbb{Q}_{p}\right):=\mathrm{H}_{\mathrm{dR}}^{q}(Y / \mathbb{Q}) \otimes \mathbb{Q}_{p},
$$

come equipped with a Frobenius operator $\operatorname{Fr}_{X}$.

A classical conjecture of Katz proved by Mazur relates the $p$-adic absolute values of the eigenvalues of $\mathrm{Fr}_{X}$ to the Hodge numbers of $Y$,

$$
h^{i, q-i}:=\operatorname{dim}_{\mathbb{Q}} \mathrm{H}^{q-i}\left(Y, \Omega_{Y}^{i}\right),
$$

where $\Omega_{Y}^{i}$ denotes the bundle of differential $i$-forms on $Y$. Order the eigenvalues $\alpha_{i}$ of $\operatorname{Fr}_{X}$ so that $\operatorname{ord}_{p}\left(\alpha_{i}\right) \leq \operatorname{ord}_{p}\left(\alpha_{i+1}\right)$, and set $\beta_{i}=h^{0, q}+h^{1, q-1}+\cdots+h^{i, q-i}$.

\footnotetext{
${ }^{4}$ The results holds more generally for any algebraic variety assuming that the cohomology is taken with compact supports.
} 
Theorem 12 (Katz's conjecture [19], Mazur [22, 23]). With the notations introduced above,

$$
\begin{aligned}
& \operatorname{ord}_{p}\left(\alpha_{1}\right)+\cdots+\operatorname{ord}_{p}\left(\alpha_{t}\right) \\
& \quad \geq 0 \cdot h^{0, q}+1 \cdot h^{1, q-1}+\cdots+i h^{i, q-i}+(i+1)\left(t-\beta_{i}\right),
\end{aligned}
$$

where $\beta_{i}<t<\beta_{i+1}$.

There is a more geometric way to state theorem 12. Consider the Newton polygon ${ }^{5}$ of the characteristic polynomial $\operatorname{det}\left(\mathrm{id}-\mathrm{Fr}_{X} \cdot t\right)$ and the polygon associated to the Hodge filtration of $\mathrm{H}_{\mathrm{dR}}^{q}\left(X / \mathbb{Q}_{p}\right)$ :

$$
F^{i} \mathrm{H}_{\mathrm{dR}}^{q}\left(X / \mathbb{Q}_{p}\right):=\bigoplus_{j=i}^{q} \mathrm{H}^{q-j}\left(Y, \Omega_{Y}^{j}\right) \otimes \mathbb{Q}_{p} .
$$

Concretely, these polygons are piecewise linear functions starting at $(0,0)$ and with slopes

\begin{tabular}{r|c|c} 
& Newton polygon & Hodge polygon \\
\hline $\begin{array}{r}\text { slope } \\
\text { on the } \\
\text { interval }\end{array}$ & $\operatorname{ord}\left(\alpha_{i}\right)$ & $i$ \\
{$\left[\sum_{j=1}^{i-1} m_{j}, \sum_{j=1}^{i} m_{j}\right]$} & {$\left[\sum_{j=0}^{i-1} h^{j, q-j}, \sum_{j=0}^{i} h^{j, q-j}\right]$}
\end{tabular}

where $m_{i}$ is the multiplicity of the eigenvalue $\alpha_{i}$. Theorem 12 becomes "the Newton polygon lies above the Hodge polygon".

Example 13. Suppose $X$ is a smooth projective curve of genus $g$. Let me collect here some information on the Newton polygon of $X$ :

- By Serre duality, $h^{0,1}=h^{1,0}=g$.

- Poincaré duality implies that the Newton polygon ends at $(2 g, g)$, as the Hodge polygon;

- Let $A=\operatorname{Jac}(X)$ be the jacobian variety of $X$. For a positive integer $n$ denote by $A[n]$ the subgroup of $n$-torsion points of $A\left(\overline{\mathbb{F}}_{p}\right)$. There is an integer $0 \leq \operatorname{rk}_{p}(A) \leq g$, the $p$-rank of $A$, such that

$$
\# A[n]= \begin{cases}n^{\mathrm{rk}_{p}(A)} & \text { if } n \text { is a power of } p, \\ n^{2 g} & \text { if } n \text { is prime to } p .\end{cases}
$$

The previous information describe completely the Newton polygon of $X$ for $g=1,2$. However, starting from $g=3$ the situation is more involved (see Figure 1).

\section{Filtered isocrystals}

A strengthening of Mazur's theorem, conjectured by Fontaine [15] and proved by Faltings [12], states that the inequality (2) holds for every sub-vector space $W \subset \mathrm{H}_{\mathrm{dR}}^{q}\left(X / \mathbb{Q}_{p}\right)$ stable under the action of the Frobenius endomorphism:

$$
\sum_{i} i \operatorname{dim}_{\mathbb{Q}_{p}}\left(W^{i} / W^{i+1}\right) \leq \operatorname{ord}_{p} \operatorname{det}\left(\operatorname{Fr}_{X} \mid W\right)
$$

where $W^{i}:=F^{i} \mathrm{H}_{\mathrm{dR}}^{q}\left(X / \mathbb{Q}_{p}\right) \cap W$. Moreover, the fact that both polygons have the same end-points says that the previous inequality is an identity for $W=\mathrm{H}_{\mathrm{dR}}^{q}\left(X / \mathbb{Q}_{p}\right)$.

A filtered isocrystal over $\mathbb{Q}_{p}$ is a linear algebraic datum miming the above situation: it is a triple $\left(V, \varphi, F^{\bullet} V\right)$ made of

\footnotetext{
${ }^{5}$ Given a polynomial $f(t)=a_{0}+a_{1} t+\cdots+a_{d} t^{d}$ with coefficients in $\mathbb{Q}_{p}$, its Newton polygon is the convex hull of the points $\left(i, \operatorname{ord}_{p}\left(a_{i}\right)\right)$, i.e. the smallest convex function on the interval $[0, d]$ such that the points $\left(i, \operatorname{ord}_{p}\left(a_{i}\right)\right)$ lie above (or on) its graph.
}

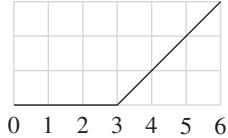

$\operatorname{rk}_{p}(A)=3$

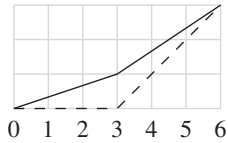

$\mathrm{rk}_{p}(A)=0$

$A$ not super-singular

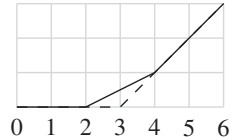

$\operatorname{rk}_{p}(A)=2$

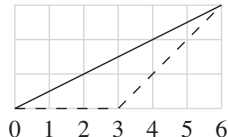

$\operatorname{rk}_{p}(A)=0$

$A$ super-singular

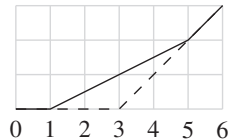

$\operatorname{rk}_{p}(A)=1$

- Newton polygon

- - Hodge polygon

Figure 1: Newton polygons for a curve of genus 3

a finite-dimensional $\mathbb{Q}_{p}$-vector space, a linear map $\varphi: V \rightarrow V$ and a decreasing filtration $F^{\bullet} V=\left(F^{i} V\right)_{i \in \mathbb{Z}}$. It is said to be weakly admissible if, for every linear subspace $W \subset V$ stable under $\varphi$,

$$
\sum_{i} i \operatorname{dim}_{\mathbb{Q}_{p}}\left(W^{i} / W^{i+1}\right) \leq \operatorname{ord}_{p} \operatorname{det}(\varphi \mid W),
$$

where $W^{i}:=F^{i} V \cap W$, with equality for $W=V .^{6}$

Weakly admissible filtered isocrystals are meant to be one of the possible $p$-adic analogues of Hodge structures. Because of the Künneth formula for cohomology, it is natural to ask whether the tensor product of weakly admissible isocrystals is weakly admissible. A partial affirmative answer was given by Lafaille [21], while the general result was proven by Faltings $[13,14]$ and Totaro [32].

\section{The role of semi-stable vector bundles}

In order to explain the approaches of Faltings and Totaro, let me consider a slightly different situation.

Let $V$ be a finite dimensional $\mathbb{Q}_{p}$-vector space, $K$ a finite extension of $\mathbb{Q}_{p}$ and $F^{\bullet} V=\left(F^{i} V\right)$ a filtration of the $K$-vector space $V \otimes_{\mathbb{Q}_{p}} K$ by $K$-vector spaces. For a $\mathbb{Q}_{p}$-linear subspace $W \subset V$ define

$$
\operatorname{deg} W=\sum_{i} i \operatorname{dim}_{\mathbb{Q}_{p}}\left(W^{i} / W^{i+1}\right),
$$

where $W^{i}:=F^{i} V \cap\left(W \otimes_{\mathbb{Q}_{p}} K\right)$.

The filtered vector space $\left(V, F^{\bullet} V\right)$ is said to be semi-stable if, for all non-zero $\mathbb{Q}_{p}$-linear subspace $W \subset V$,

$$
\mu(W):=\frac{\operatorname{deg} W}{\operatorname{dim} W} \leq \mu(V):=\frac{\operatorname{deg} V}{\operatorname{dim} V} .
$$

Example 14. Suppose $V=\mathbb{Q}_{p}^{2}$. A point $x \in \mathbb{P}^{1}\left(\overline{\mathbb{Q}}_{p}\right)$ corresponds to a vector line $L \subset V \otimes_{\mathbb{Q}_{p}} K$ for a suitable finite extension $K$ of $\mathbb{Q}_{p}$. The filtration

$$
F^{2} V:=0 \quad \subset \quad F^{1} V:=L \quad \subset \quad F^{0} V:=V \otimes_{\mathbb{Q}_{p}} K
$$

satisfies $\mu(V)=\frac{3}{2}$ and $\mu(W)=2-\operatorname{dim}_{K} L \cap\left(W \otimes_{\mathbb{Q}_{p}} K\right)$ for every $\mathbb{Q}_{p}$-vector line $W \subset V$. In particular $\left(V, F^{\bullet} V\right)$ is semistable if and only if the line $L$ is not defined over $\mathbb{Q}_{p}$.

The set of semi-stable filtrations on $V$ is Drinfeld's upper half-plane $\Omega_{\mathbb{Q}_{p}}^{1}:=\mathbb{P}^{1}\left(\overline{\mathbb{Q}}_{p}\right) \backslash \mathbb{P}^{1}\left(\mathbb{Q}_{p}\right)$. It owes its name to Poincaré's upper-half plane $\mathfrak{h}=\{z \in \mathbb{C}: \operatorname{Im} z>0\}$ which can be seen as the "upper-half" of $\mathbb{P}^{1}(\mathbb{C}) \backslash \mathbb{P}^{1}(\mathbb{R})$.

\footnotetext{
${ }^{6}$ Actually this definition is too restrictive, as one has to let the filtration $F^{\bullet} V$ to be defined only on a finite extension of $\mathbb{Q}_{p}$.
} 
To have a picture in mind, Drinfeld's upper half-plane (or better the Berkovich analytic space attached to it) retracts onto the Bruhat-Tits building of $\mathrm{SL}_{2}\left(\mathbb{Q}_{p}\right)$; see Figure 2.

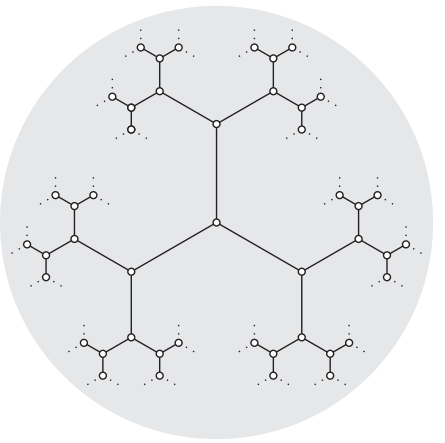

Figure 2: The Bruhat-Tits building of $\mathrm{SL}_{2}\left(\mathbb{Q}_{p}\right)$ for $p=2$

Suppose that $K$ is a Galois extension of $\mathbb{Q}_{p}$ and consider the filtrations $F_{1}^{\bullet} V, \ldots, F_{n}^{\bullet} V$ conjugated to $F^{\bullet} V$ under the action of the Galois group $\operatorname{Gal}\left(K / \mathbb{Q}_{p}\right)$. Faltings' idea is to associate to these filtrations a vector bundle $E(V)$ on a compact Riemann surface $Y$ such that $E(V)$ is semi-stable (as a vector bundle) if and only if the filtered vector space $\left(V, F^{\bullet} V\right)$ is. Since this construction is compatible with tensor product, one draws the result thanks to the theorem of NarasimhanSeshadri.

The proof of Totaro is again based on the tensor product of semi-stable bundles, but instead of relying on the "analytic" result of Narasimhan-Seshadri, it follows the "algebraic" approach of Ramanan-Ramanathan.

Totaro characterizes semi-stability of filtrations in terms of Geometric Invariant Theory. It is just a matter of unwinding the definitions: the reader familiar with GIT recognizes the quantity appearing in (3) as Mumford's $\mu$-coefficient in the case of filtrations [24, §4.4]. Then he uses RamananRamanathan's dichotomy to derive the wanted inequality.

\section{Hermitian vector bundles on arithmetic curves}

\section{Euclidean lattices}

An Euclidean lattice of rank $n$ is a finitely generated subgroup $\Gamma$ of $\mathbb{R}^{n}$ such that $\mathbb{R}^{n} / \Gamma$ is compact. On $\mathbb{R}^{n} / \Gamma$ there is a unique measure $\mu_{\Gamma}$ such that, for all continuous functions $f: \mathbb{R}^{n} \rightarrow \mathbb{R}$ with compact support,

$$
\int_{\mathbb{R}^{n}} f(x) d \lambda(x)=\int_{\mathbb{R}^{n} / \Gamma} \tilde{f}(\xi) d \mu_{\Gamma}(\xi),
$$

where $\lambda$ is Lebesgue measure on $\mathbb{R}^{n}$ and $\tilde{f}(\xi)=\sum_{\Gamma+x=\xi} f(x)$. The volume of $\mathbb{R}^{n} / \Gamma$ with respect to this measure is called the covolume of $\Gamma$. If $\gamma_{1}, \ldots, \gamma_{n}$ is a basis of $\Gamma$, the covolume $\operatorname{covol}(\Gamma)$ can be computed as $\left|\operatorname{det}\left(\gamma_{1}, \ldots, \gamma_{n}\right)\right|$.

There is a more intrinsic definition of a Euclidean lattice: it is a couple $\bar{E}=\left(E,\|\cdot\|_{E}\right)$ made of a free abelian group $E$ of finite rank together with a hermitian norm $\|\cdot\|_{E}$ on $E \otimes_{\mathbb{Z}} \mathbb{R}$. Following conventions that have become usual in Arakelov geometry, define:

$$
\begin{array}{lr}
\text { the rank of } \bar{E} & \operatorname{rk}(\bar{E}):=\operatorname{dim}_{\mathbb{R}} E \otimes_{\mathbb{Z}} \mathbb{R}, \\
\text { the degree of } \bar{E} & \widehat{\operatorname{deg}}(\bar{E})=-\log \operatorname{covol}(E), \\
\text { the slope of } \bar{E} & \hat{\mu}(\bar{E})=(\mathrm{rk} \bar{E})^{-1} \widehat{\operatorname{deg}} \bar{E} .
\end{array}
$$

\section{Diophantine approximation}

Before diving into the analogy with vector bundles, let me explain how these concepts arise in Diophantine approximation.

Theorem 15 (Thue [31]). Let $\alpha \in \mathbb{R}$ be an algebraic number of degree $d \geq 2$. Given $\varepsilon>0$ there are only finitely many rational numbers $p / q \in \mathbb{Q}$ such that

$$
\left|\alpha-\frac{p}{q}\right|<\frac{1}{q^{\frac{d}{2}+1+\varepsilon}},
$$

where $(p, q)=1$ and $q>0$.

The exponent $\frac{d}{2}+1$ has been successively sharpened by Siegel, Dyson and Roth respectively to $2 \sqrt{d}, \sqrt{2 d}$ and 2, the latter being optimal because of Dirichlet's theorem on approximation of real numbers. However all these proofs follow the scheme of Thue's argument, which can be roughly split in four steps:

1. Construct a polynomial $f$ in $n$ variables with small integral coefficients vanishing "as much as possible" on the point $(\alpha, \ldots, \alpha)$.

2. Prove that the polynomial $f$ does not vanish too much on $n$-tuples $x=\left(x_{1}, \ldots, x_{n}\right)$ made of rational approximations. Or better said some derivative $g$ of $f$ does not vanish at $x$.

3. Bound from above $|g(x)|$ in terms of the order of vanishing of $g$ at $(\alpha, \ldots, \alpha)$ by looking at its Taylor expansion.

4. Bound from below $|g(x)|$ by using that a positive integer is $\geq 1$.

When there are too many good approximations of $\alpha$ to exist, bounds in 3 and 4 are in contradiction.

In his Bourbaki report on the work of Masser and Wüstholz on periods and isogenies of abelian varieties (having among its consequences Mordell's Conjecture, by the time already a theorem of Faltings), Bost reinterprets in terms of Euclidean lattices the preceding steps.

The point is to use a simple fact on the behaviour of slopes with respect to linear maps:

Lemma 16 (Slopes inequality). Let $\bar{E}, \bar{F}$ be Euclidean lattices and $\varphi: E \rightarrow F$ an injective map of abelian groups. Then,

$$
\hat{\mu}(\bar{E}) \leq \hat{\mu}_{\max }(\bar{F})+\log \|\varphi\|_{\text {sup }},
$$

where $\hat{\mu}_{\max }(\bar{F})$ is the maximum of the slopes of sub-lattices of $\bar{F}$ and $\|\varphi\|_{\text {sup }}$ is the operator norm of $\varphi$.

Thue's four steps argument is translated as follows. Step 2, which is the one with geometric content, corresponds to the injectivity ${ }^{7}$ of the map $\varphi$. Step 3 corresponds to the upper bound of $\|\varphi\|_{\text {sup }}$ and step 4 to bounding the slopes of $\bar{E}$ and $\bar{F}$.

A step that seems gone missing is the first one, which is indeed a little different. Instead of picking a particular polynomial, the slopes inequality allows to consider the whole space of polynomials with the wanted vanishing property. In the concrete case of Masser and Wüstholz this permitted Bost to replace fine considerations on theta functions by geometric arguments of Moret-Bailly.

\section{Semi-stable lattices}

A Euclidean lattice $\bar{E}$ is said to be semi-stable if $\hat{\mu}(\bar{F}) \leq \hat{\mu}(\bar{E})$ for all non-zero sub-lattices $F \subset E$ with the induced Euclidean norm.

\footnotetext{
${ }^{7}$ In this situation $\varphi$ is the evaluation map of a polynomial at $\alpha$ (or better, some truncated Taylor expansion of $f$ around $\alpha$ ).
} 
Question (Bost). Is the tensor product ${ }^{8}$ of semi-stable lattices semi-stable?

Results towards a positive answer to this question have been proved by André [1], Bost, de Shalit-Parzanovski, Chen [7] and Bost-Künneman [6]. The best available result is the following:

Theorem 17 (Bost-Chen [5]). Let $\bar{E}, \bar{F}$ be semi-stable Euclidean lattices. Then,

$$
\hat{\mu}_{\max }(\bar{E} \otimes \bar{F}) \leq \hat{\mu}(\bar{E})+\hat{\mu}(\bar{F})+\frac{1}{2} \min \{\ell(\operatorname{rk} E), \ell(\operatorname{rk} F)\},
$$

where, for an integer $n \geq 2, \ell(n)=\sum_{i=2}^{n} \frac{1}{i} \leq \log n$.

Furthermore, if $\operatorname{rk} E \cdot \operatorname{rk} F \leq 9$, then $\bar{E} \otimes \bar{F}$ is semi-stable.

\section{Analogy with the projective line?}

The field of rational numbers ressembles somehow to the field of rationals functions in one variable. This analogy works by making correspond points of $\mathbb{P}^{1}$ to equivalence classes of absolute values on $\mathbb{Q}$. Stressing this point of view, the avatar over $\mathbb{Q}$ of vector bundles are Euclidean lattices.

Nonetheless the theory of vector bundles on $\mathbb{P}^{1}$ is rather poor: a vector bundle on $\mathbb{P}^{1}$ is a direct sum of line bundles ${ }^{9}$, and semi-stable vector bundles are all of the form $O(d)^{\oplus n}$.

This is not at all the situation for Euclidean lattices: the set of isomorphism classes of Euclidean lattices of rank $n$ is the double quotient $\mathrm{GL}_{n}(\mathbb{Z}) \backslash \mathrm{GL}_{n}(\mathbb{R}) / \mathrm{O}_{n}(\mathbb{R})$, where $\mathrm{O}_{n}(\mathbb{R})$ is the group of orthogonal matrices of size $n$. Moreover, if one associates to a point $\tau$ in the upper half plane the lattice generated by 1 and $\tau$, then the region of semi-stable lattices of rank two is the closed exterior of the Ford circles in the strip $0<\operatorname{Im} \tau \leq 1[1]$.

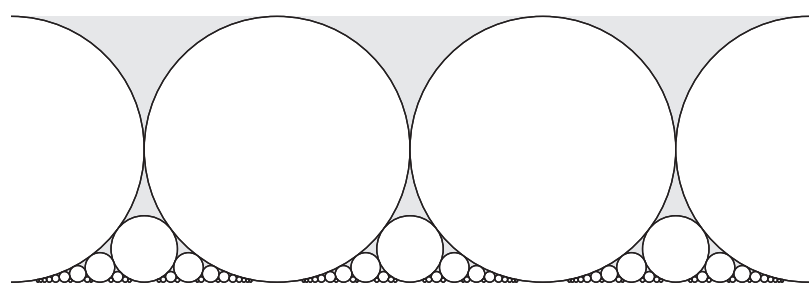

Figure 3: The exterior of Ford circles

\section{Irreducible Euclidean lattices}

Given a Euclidean lattice $\bar{E}$, denote by $\operatorname{Aut}(\bar{E})$ the group of elements $g \in \mathrm{GL}(E)$ respecting the Euclidean norm: it is a finite group. A Euclidean lattice $\bar{E}$ is said to be irreducible if the representation $\operatorname{Aut}(\bar{E}) \rightarrow \operatorname{GL}\left(E \otimes_{\mathbb{Z}} \mathbb{Q}\right)$ is.

Example 18. Consider the real plane

$$
H=\left\{\left(x_{1}, x_{2}, x_{3}\right) \in \mathbb{R}^{3}: x_{1}+x_{2}+x_{3}=0\right\},
$$

\footnotetext{
${ }^{8}$ The Euclidean norm on the tensor product is defined as follows: given Euclidean spaces $V, W$ with scalar products $\langle\cdot, \cdot\rangle_{V}$ and $\langle\cdot, \cdot\rangle_{W}$, the tensor product $V \otimes_{\mathbb{R}} W$ is given the structure of Euclidean space by defining

$$
\left\langle v \otimes w, v^{\prime} \otimes w^{\prime}\right\rangle_{V \otimes W}=\left\langle v, v^{\prime}\right\rangle_{V} \cdot\left\langle w, w^{\prime}\right\rangle_{W}
$$

${ }^{9}$ In the modern literature, this theorem is sometimes referred to as the Grothendieck-Birkhoff decomposition. However, it is easily deduced from the Elementary Divisors theorem for the ring $k\left[t, t^{-1}\right]$, due to DedekindWeber [9], or its complex analytic analogue discovered by Birkhoff [4].
}

inheriting the standard scalar product of $\mathbb{R}^{3}$. Consider the lattice $E=H \cap \mathbb{Z}^{3}$. Identifying $H$ with the standard Euclidean plane $\mathbb{R}^{2}, E$ corresponds to the vertices of a planar tessellation by equilateral triangles.

The group of isometries of $E$ is the group of permutations on 3 elements, which acts irreducibly on $H$.

Other examples of irreducible Euclidean lattices come from reductive groups! Indeed, over an algebraically closed field, reductive groups are classified by a combinatorial datum called the root system. Simple Lie algebras correspond to irreducible root systems, which give rise to irreducible Euclidean lattices: the example above is the root system of $\mathrm{SL}_{3}$.

Theorem 19 (Bost). 1. An irreducible Euclidean lattice is semi-stable.

2. The tensor product of irreducible Euclidean lattices is irreducible.

The first statement follows from the existence of the Harder-Narasimhan filtration: there is a sub-lattice $\bar{E}_{\max }$ realizing the biggest slope and which is maximal for this property. By maximality, it is unique hence stable under the action of $\operatorname{Aut}(\bar{E})$. Therefore, if $\bar{E}$ is irreducible, $\bar{E}=\bar{E}_{\text {max }}$.

The second is a straightforward consequence of the following classical result:

Theorem 20. Let $k$ be a field of characteristic 0 and $V_{i}$ an irreducible representation of a group $G_{i}$ over $k(i=1,2)$. Then $V_{1} \otimes_{k} V_{2}$ is an irreducible representation of $G_{1} \times G_{2}$.

Theorem 20 follows from the formula

$$
\operatorname{End}_{G_{1} \times G_{2}}\left(V_{1} \otimes_{k} V_{2}\right)=\operatorname{End}_{G_{1}}\left(V_{1}\right) \otimes \operatorname{End}_{G_{2}}\left(V_{2}\right)
$$

and Schur's Lemma: if $k$ is algebraically closed and $V$ is an irreducible representation of a group $G$, then $\operatorname{End}_{G}(V)=k$.

Formula (4) is a special case of Jacobson's density theorem. Another proof, similar to that of Chevalley's theorem, goes as follows. One may assume that $G_{1}$ and $G_{2}$ are reductive groups and the ground field is $\mathbb{C}$. For $i=1,2$ let $K_{i}$ be a Zariski-dense compact subgroup of $G_{i}$. Write a $G$-equivariant endomorphism $\varphi$ of $V_{1} \otimes_{k} V_{2}$ as

$$
\varphi=\sum_{\lambda=1}^{N} \alpha_{1 \lambda} \otimes \alpha_{2 \lambda},
$$

with $\alpha_{i \lambda} \in \operatorname{End}\left(V_{i}\right)$. By $G$-equivariance of $\varphi$ and Fubini's theorem:

$$
\begin{aligned}
\varphi\left(x_{1} \otimes x_{2}\right) & =\int_{K_{1} \times K_{2}} g \varphi\left(g^{-1}\left(x_{1} \otimes x_{2}\right)\right) d \mu_{1} \otimes d \mu_{2}(g) \\
& =\sum_{\lambda=1}^{N} \tilde{\alpha}_{1 \lambda} \otimes \tilde{\alpha}_{2 \lambda}\left(x_{1} \otimes x_{2}\right),
\end{aligned}
$$

where $\mu_{i}$ is the Haar measure of total mass 1 on $K_{i}$ and

$$
\tilde{\alpha}_{i \lambda}(x):=\int_{K_{i}} g_{i} \alpha_{i \lambda}\left(g_{i}^{-1} x_{i}\right) d \mu_{i}\left(g_{i}\right),
$$

is $G_{i}$-equivariant. 
where $\mu_{i}$ is the Haar measure of total mass 1 on $K_{i}$ and

$$
\tilde{\alpha}_{i \lambda}(x):=\int_{K_{i}} g_{i} \alpha_{i \lambda}\left(g_{i}^{-1} x_{i}\right) d \mu_{i}\left(g_{i}\right),
$$

is $G_{i}$-equivariant.

\section{Ramanan-Ramanathan method, again}

Chen translated the argument of Ramanan-Ramanathan, as elaborated by Totaro, in the context of Arakelov geometry in order to give the following bound:

$$
\hat{\mu}_{\max }\left(\bar{E}_{1} \otimes \cdots \otimes \bar{E}_{n}\right) \leq \sum_{i=1}^{n}\left(\hat{\mu}\left(\bar{E}_{i}\right)+\log \mathrm{rk} E_{i}\right) .
$$

The error term is Chen's inequality comes for a very simple reason: the operator norm of the projection $V^{\otimes \operatorname{dim} V} \rightarrow \operatorname{det} V$ is $\operatorname{dim} V !$.

\section{References}

[1] Y. André. On nef and semistable Hermitian lattices, and their behaviour under tensor product. Tohoku Math. J. (2), 63(4):629-649, 2011.

[2] V. Balaji, P. Deligne, and A. J. Parameswaran. On complete reducibility in characteristic p. L'épijournal de Géométrie Algébrique, Volume 1, 2017.

[3] V. Balaji and A. J. Parameswaran. Tensor product theorem for Hitchin pairs - an algebraic approach. Ann. Inst. Fourier (Grenoble), 61(6):2361-2403 (2012), 2011.

[4] G. D. Birkhoff. A theorem on matrices of analytic functions. Math. Ann., 74(1):122-133, 1913.

[5] J.-B. Bost and H. Chen. Concerning the semistability of tensor products in Arakelov geometry. J. Math. Pures Appl. (9), 99(4):436-488, 2013.

[6] J.-B. Bost and K. Künnemann. Hermitian vector bundles and extension groups on arithmetic schemes. I. Geometry of numbers. Adv. Math., 223(3):987-1106, 2010.

[7] H. Chen. Maximal slope of tensor product of Hermitian vector bundles. J. Algebraic Geom., 18(3):575-603, 2009.

[8] C. Chevalley. Théorie des groupes de Lie. Tome III. Théorèmes généraux sur les algèbres de Lie. Actualités Sci. Ind. no. 1226. Hermann \& Cie, Paris, 1955.

[9] R. Dedekind and H. Weber. Theorie der algebraischen Functionen einer Veränderlichen. J. Reine Angew. Math., 92:181-290, 1882.

[10] P. Deligne. Semi-simplicité de produits tensoriels en caractéristique p. Invent. Math., 197(3):587-611, 2014.

[11] M. Demazure and P. Gabriel. Groupes algébriques. Tome I: Géométrie algébrique, généralités, groupes commutatifs. Masson \& Cie, Éditeur, Paris; North-Holland Publishing Co., Amsterdam, 1970. Avec un appendice it Corps de classes local par Michiel Hazewinkel.

[12] G. Faltings. Crystalline cohomology and $p$-adic Galois-representations. In Algebraic analysis, geometry, and number theory (Baltimore, $M D, 1988$ ), pages 25-80. Johns Hopkins Univ. Press, Baltimore, MD, 1989.

[13] G. Faltings. Mumford-Stabilität in der algebraischen Geometrie. In Proceedings of the International Congress of Mathematicians, Vol. 1, 2 (Zürich, 1994), pages 648-655. Birkhäuser, Basel, 1995.

[14] G. Faltings and G. Wüstholz. Diophantine approximations on projective spaces. Invent. Math., 116(1-3):109-138, 1994.

[15] J.-M. Fontaine. Modules galoisiens, modules filtrés et anneaux de Barsotti-Tate. In Journées de Géométrie Algébrique de Rennes. (Rennes, 1978), Vol. III, volume 65 of Astérisque, pages 3-80. Soc. Math. France, Paris, 1979.

[16] J. Giraud, A. Grothendieck, S. L. Kleiman, M. Raynaud, and J. Tate. Dix exposés sur la cohomologie des schémas, volume 3 of Advanced Studies in Pure Mathematics. North-Holland Publishing Co., Amsterdam; Masson \& Cie, Editeur, Paris, 1968.
[17] D. Hilbert. Über die vollen Invariantensysteme. Math. Ann., 42(3):313-373, 1893.

[18] S. Ilangovan, V. B. Mehta, and A. J. Parameswaran. Semistability and semisimplicity in representations of low height in positive characteristic. In A tribute to C. S. Seshadri (Chennai, 2002), Trends Math., pages 271-282. Birkhäuser, Basel, 2003.

[19] N. M. Katz. On a theorem of Ax. Amer. J. Math., 93:485-499, 1971.

[20] G. R. Kempf. Instability in invariant theory. Ann. of Math. (2), 108(2):299-316, 1978.

[21] G. Laffaille. Groupes $p$-divisibles et modules filtrés: le cas peu ramifié. Bull. Soc. Math. France, 108(2):187-206, 1980.

[22] B. Mazur. Frobenius and the Hodge filtration. Bull. Amer. Math. Soc., 78:653-667, 1972.

[23] B. Mazur. Frobenius and the Hodge filtration (estimates). Ann. of Math. (2), 98:58-95, 1973.

[24] D. Mumford. Geometric invariant theory. Ergebnisse der Mathematik und ihrer Grenzgebiete, Neue Folge, Band 34. Springer-Verlag, Berlin-New York, 1965

[25] M. S. Narasimhan and C. S. Seshadri. Stable and unitary vector bundles on a compact Riemann surface. Ann. of Math. (2), 82:540-567, 1965

[26] S. Ramanan and A. Ramanathan. Some remarks on the instability flag. Tohoku Math. J. (2), 36(2):269-291, 1984

[27] G. Rousseau. Instabilité dans les espaces vectoriels. In Algebraic surfaces (Orsay, 1976-78), volume 868 of Lecture Notes in Math., pages 263-276. Springer, Berlin-New York, 1981.

[28] J.-P. Serre. Cours d'arithmétique, volume 2 of Collection SUP: “Le Mathématicien”. Presses Universitaires de France, Paris, 1970

[29] J.-P. Serre. Sur la semi-simplicité des produits tensoriels de représentations de groupes. Invent. Math., 116(1-3):513-530, 1994.

[30] J.-P. Serre. 1998 Moursund Lectures at the University of Oregon. arXiv:math/0305257 May 2003.

[31] A. Thue. Über Annäherungswerte algebraischer Zahlen. J. Reine Angew. Math., 135:284-305, 1909.

[32] B. Totaro. Tensor products in p-adic Hodge theory. Duke Math. J., 83(1):79-104, 1996.

[33] H. Weyl. Theorie der Darstellung kontinuierlicher halb-einfacher Gruppen durch lineare Transformationen. I. Math. Z., 23(1):271-309, 1925

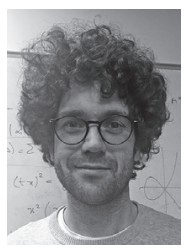

Marco Maculan [marco.maculan@imjprg.fr] received his PhD in Paris in 2012. He is now a Maître de Conférences at Université Pierre et Marie Curie. His field of interest is arithmetic geometry, especially in connection with p-adic analysis and algebraic groups. 